\title{
PEQUEÑOS TOQUES HACEN GRANDES RASGOS: ANÁLIISIS DE LOS DETERMINANTES DE LAS MICRO, PEQUEÑAS Y MEDIANAS EMPRESAS, PYME
}

\author{
THE BIG PICTURE IS MADE OF MANY SMALL PIECES: ANALYSIS \\ OF THE DETERMINANTS OF MICRO, SMALL AND MEDIUM-SIZED \\ ENTERPRISES, SMES
}

\section{Jairo Rivera. ${ }^{1,2}$}

Este trabajo analiza los determinantes de las micro, pequeñas y medianas empresas, PYME. Inicialmente se aborda las definiciones de PYME en distintos bloques regionales y la adoptada por el Ecuador. Posteriormente, se desarrolla la sección empírica utilizando datos correspondientes al Censo Nacional Económico 2010 (CENEC). En la estimación se realiza un análisis cuantitativo sobre las similitudes y diferencias de la clasificación de PYME mediante el uso de personal ocupado y ventas, a nivel general y sectorial. Adicionalmente se plantea un modelo probabilístico tomando en cuenta las variables geográficas, sectoriales y administrativas. Los resultados indican que hay diferencias entre el criterio utilizado y que pertenecer al sector comercio o servicios aumenta la probabilidad de ser PyME, mientras que formalizar la parte administrativa, con RUC y registros administrativos, reduce su probabilidad.

PALABRAS CLAVE: PyME, análisis cuantitativo, modelo probabilístico, Ecuador
This paper analyzes the determinants of small and medium-sized enterprises, SMEs. Initially, there are examined the definitions of SMEs in different regional blocs and the one that was adopted by Ecuador. Subsequently, the empirical section is developed using data for the National Economic Census 2010 (CENEC). In estimating there is performed a quantitative analysis of the similarities and differences in the classification of SMEs by using employed persons and sales, general and sectoral level. In addition, there is developed a probabilistic model taking into account geographical, sectoral and administrative variables. The results indicate that there are differences between the criteria used, the probability of being SMEs increases when the enterprise is in trade or services sector, while formalizing the administration, with RUC and administrative records, reduce its chance.

KEYWORDS: SME, quantitativeanalysis, probabilisticmodel, Ecuador. 


\section{Introducción}

La micro, pequeña y mediana empresa, PyME, tiene un rol fundamental en el desarrollo social y económico de los países (Ayyagari,Demirgüc-Kunt, y Maksimovic, 2011). El concepto de PyME no es uniforme ni estático alrededor del mundo. Ante ello, los distintos bloques regionales han desarrollado sus propias concepciones, existiendo diversidad de elementos que se toman en cuenta para clasificar a las empresas y que sus resultados dependen de dichos criterios. Las variables clave que han sido utilizadas son: el número de trabajadores o personal ocupado, el valor de las ventas $\mathrm{y}$ el valor de los activos. Adicionalmente, desde otros enfoques, existen propuestas de categorizar a las empresas de forma sectorial o por fórmulas.

Los países desarrollados, miembros de la Organización para la Cooperación y el Desarrollo Económico-OECD- (por sus siglas en inglés), manejan una definición de PyME basada en el número de trabajadores. Con ello, para la OECD, una PyME es aquella que posee hasta 250 trabajadores. Dentro de esta clasificación se puede subdividir a las microempresas, que están compuestas hasta por 10 trabajadores, y las pequeñas empresas, que son aquellas que tienen hasta 50 trabajadores (OECD, 2005a). Estados Unidos maneja una definición de PyME de acuerdo a dos criterios: los ingresos anuales o el número de trabajadores. La característica particular de la categorización de pequeña y mediana empresa en este país es que toma en cuenta el sector industrial de actividad económica. Para ejemplificar se puede decir que el sector agrícola tiene pequeñas empresas si posee hasta 500 trabajadores, mientras que en el sector de minería el rango va desde 250 a 1500 trabajadores (SBA, 2016). Este ajuste sectorial tiene la potencialidad de expresar las características propias de cada sector económico y alejarse en cierto sentido de una estandarización que homogeneiza a la economía. A pesar de la existencia de dichas especificidades, en general se podría definir a una PyME como aquella que tiene hasta 500 trabajadores (OECD, 2007).

La Unión Europea desde el año 2003 opera con un concepto de pequeñas y medianas empresas basado en dos elementos: volumen de negocios o balance general y número de trabajadores. Ante ello, las PyME se definen como aquellas empresas que emplean a menos de 250 personas y cuyo volumen de negocios anual o cuyo balance general anual no exceda del 50 millones de euros (EuropeanUnion, 2015). El Mercado Común del Sur-MERCOSUR- desde el año 1992 maneja un concepto de pequeñas y medianas empresas de acuerdo a tres elementos: número de trabajadores, valor bruto de las ventas anuales y el coeficiente de tamaño. El coeficiente de tamaño es un elemento innova- dor que relaciona el número de trabajadores y las ventas de una empresa determinada con sus valores referenciales (MERCOSUR, 1993). Con ello, las PyME se definen como empresas de hasta 299 trabajadores, con valores brutos de ventas de hasta 10 millones de dólares y un coeficiente de tamaño de hasta 10. La Comunidad Andina de Naciones -CAN- expidió en el año 2008 la Decisión 702, en ella se define a una pequeña y mediana empresa en base a dos características: número de trabajadores y valor bruto de las ventas anuales. En la decisión se indica que se otorga preferencia a los valores de ventas. Entonces, una PyME es aquella que tiene menos de 200 trabajadores y ventas menores a 5 millones (CAN, 2008).

El Ecuador desde el año 2010 decidió adoptar formalmente la Decisión 702 de la CAN. Esto lo realizó mediante el Código Orgánico de la Producción, Comercio e Inversiones (COPCI). Con ello, son dos variables las que definen una PYME: el personal ocupado y el valor bruto de las ventas. La PyME en el país tiene un rol fundamental en la producción y empleo nacional (Araque, 2018), representando alrededor de noventa y nueve de cada cien empresas (Sarango, Álvarez-García y Del Río-Rama, 2018). En ese sentido, la economía ecuatoriana, al igual que las latinoamericanas, depende estructuralmente del desarrollo de dicho sector (Perez y Stumpo, 2000) con aporta fundamentalmente en la construcción del tejido empresarial del país (Araque y Argüello, 2015).

De la revisión de la literatura se encuentra que la definición de PyME se encuentra en debate sobre si toma en cuenta las disparidades sectoriales, geográficas, administrativas, y financieras de las pequeñas y medianas empresas. En esa línea, hay autores progresistas que critican la definición estándar de PyME. Argumentan que el entorno económico, financiero $y$ empresarial ha cambiado paulatinamente en el tiempo, por lo que se hace necesaria una definición que incorpore nuevos elementos. Por un lado, se critica las categorizaciones de PyME que se aplican a más de un país, ya que se considera que por sus particularidades cada país debería tener una concepción propia. Una posible consecuencia de dicha omisión es la distorsión en la asignación de recursos. Ante ello plantean el uso de máximos y mínimos de acuerdo al producto interno bruto por persona, es decir, un país con un PIB por habitante de mayor magnitud, tendrá una relación directa con una PyME de mayor tamaño (Gibson y Van der Vaart, 2008).

De igual manera, otras investigaciones consideran que es fundamental la inclusión de elementos relacionados con el clima de negocios. En ese sentido, plantean la incorporación del grado de formalidad, barreras de entrada, acceso a financiamiento y disponibilidad de información (Ayyagari, Thorsten,y Demir- 
güc-Kunt, 2005). Además, existen estudios que se sustentan en una adecuada disponibilidad de información y promueven la inclusión de cinco factores primarios para la definición de PyME: tamaño medio de las empresas, grado de competencia en una industria, costos de inicio y las barreras de entrada, distribución de las empresas por tamaño y proporción de pequeñas empresas en los contratos públicos (SBA, 2009). Igualmente, existen autores que promocionan la introducción de elementos que permitan diferenciar a las PyME mediante factores legales y la proporción de capital autónomo ya que eso determina la toma de decisiones (OECD, 2005b). En la misma línea hay quienes proponen la inclusión de elementos que exhiban la propiedad de recursos propios, sociedades o vínculos empresariales (EuropeanUnion, 2015).

En resumen, se puede apreciar desde la literatura de pequeñas y medianas empresas que no hay consenso en su definición. La estandarización, si bien bastante útil para homogeneizar indicadores, podría tener un efecto adverso en la diversidad de las empresas. Asimismo, un resultado robusto en las investigaciones mencionadas es que los elementos utilizados para definir a una PyME determinan sus resultados ya que cada categoría incorpora elementos particulares al sector (Briozzo, Vigier y Martínez, 2016). Ante ello, esta investigación tiene como objetivo principal el analizar los determinantesde la PyME en Ecuador, un enfoque poco examinado.

\section{Metodología}

Existen dos momentos metodológicos claramente definidos para el documento de investigación. El primero consiste en un análisis cuantitativo sobre las similitudes y diferencias de la clasificación de PYME mediante el uso de personal ocupado y ventas a nivel general y sectorial. En este proceso se analiza inicialmente las diferencias entre criterios a nivel sectorial. Posteriormente se examina transversalmente por tamaño de empresa y sector, la relación con variables geográficas y administrativas. Es necesario indicar que se eliminan los registros sin información de trabajadores o ventas. El método principal del análisis es cuantitativo y se basa inicialmente en correlaciones. Para determinar si existe dependencia entre las variables se realiza el contraste de Pearson.

El segundo momento plantea realizar un modelo probabilísticopara determinar la probabilidad de pertenecer a la clasificación de la CAN tomando en cuenta las variables geográficas, sectoriales y administrativas. La utilidad de este modelo está en ayudar a dilucidar que variables, adicionales a las ya establecidas por la Decisión 702, tienen efectos significativos en la categorización de pequeñas y medianas
empresas.El modelo probabilístico toma en cuenta las PyME por valor de ventas, debido a las recomendaciones de la CAN y la literatura, con sus características geográficas, sectoriales y administrativas. Con ello, el modelo estimado se muestra en (1):

$\mathrm{Y}_{\mathrm{i}}=\rho^{*}$ Características geográficas $_{\mathrm{i}}+\alpha *$ Características $^{*}$ sectoriales $_{i}+\beta *$ Características administrativas $_{i}+\varepsilon_{i}$

Donde Y es una variable dummy que toma el valor de 1 si la empresa es micro, pequeña o mediana empresa y o si no lo es. Entre las variables geográficas está la región natural donde se ubica la empresa, siendo 1 si la empresa está en la Sierra, Costa o Amazonía y o la región Insular; entre las características sectoriales se encuentra el sector de la actividad económica de la empresa, siendo 1 comercio o servicios y o el sector de manufactura. Por último, las variables administrativas se refieren a variables binarias del año de creación, género del gerente, disponibilidad de RUC, registros contables, capacitación, internet y afiliación a gremios.

La principal fuente de información de la presente investigación es el Censo Nacional Económico-CENEC- 2010, desarrollado por el Instituto Nacional de Estadística y Censos del Ecuador (INEC), el cual dispone de datos de las unidades económicas que conforman el sector productivo, sus características principales, y su actividad económica (Massón-Guerra \&Ortín-Ângel 2019).

El censo económico ecuatoriano previo al del 2010 se produjo a inicios de los años 80, por lo que la relevancia del Censo Nacional Económico vigente está en poseer información actualizada de los establecimientos económicos a nivel nacional.Los establecimientos censados comprenden las empresas, los establecimientos visibles, y los locales auxiliares, con datos desagregados hasta nivel cantonal (Alvarado, Peñarreta, Armas, Alvarado, 2017). Los sectores que se incluyeron en el CENEC son los siguientes: manufactura, construcción, comercio, restaurantes y hoteles, transporte y comunicaciones, intermediación financiera, servicios inmobiliarios y a las empresas, administración pública, educación, salud, servicios sociales y personales (es necesario indicar que no toma en cuenta el sector agrícola) (INEC, 2011).

\section{Resultados}

A continuación, se presenta la descripción de los datos del censo de forma general.

\section{Análisis descriptivo}

Por un lado, se puede afirmar que el número de establecimientos a nivel nacional es 511.130. Las cinco provincias con mayor cantidad de establecimientos son: Guayas (23.4\%), 
Pichincha (22.2\%), Azuay (7.1\%), Manabí (6.5\%) y Tungurahua (4.9\%). Las empresas se encuentran distribuidas sectorialmente en: Comercio (53.9\%), Servicios (36.3\%), Manufactura (9.6\%), $\mathrm{y}$ otros $(0.2 \%)$.

De acuerdo al año de constitución se aprecia que cerca de un $22 \%$ se crearon hasta el año 1999, en el periodo 2000-2005 se constituyeron un cuarto, y en el último quinquenio se crearon cerca de $53 \%$ de las empresas. Alrededor de un $67.4 \%$ posee Registro Único de Contribuyentes, cerca de $12.5 \%$ manejan registros contables, 51.6\% tienen gerentes de sexo masculino, $4.1 \%$ gastaron en formación o capacitación, $13.2 \%$ están afiliadas a un gremio, y el 11\% usan internet.

A continuación, se presenta la tabla 1 de estadísticas descriptivas de las empresas ecuatorianas:

Tabla 1 Estadísticas descriptivas

\begin{tabular}{cc} 
& Porcentaje de establecimientos \\
\hline Comercio & 53.9 \\
Servicios & 36.3 \\
Manufactura & 9.8 \\
\hline Posee RUC & 78.0 \\
Tiene registros contables & 67.4 \\
Gerente masculino & 12.5 \\
\hline Gastos en capacitación & 51.6 \\
Afiliación a gremios & 4.1 \\
Uso de internet & 13.2 \\
Nota. Cada categoría mide un porcentaje independiente.
\end{tabular}

En referencia a las variables de mayor interés del estudio, es decir aquellas utilizadas por la CAN para definir una pequeña y mediana, número de trabajadores y valor bruto de ven- tas, a continuación se presentan las figuras 1 y 2, en donde se aprecia una ligera distinción de la estructura de las empresas de acuerdo al criterio usado en su definición.

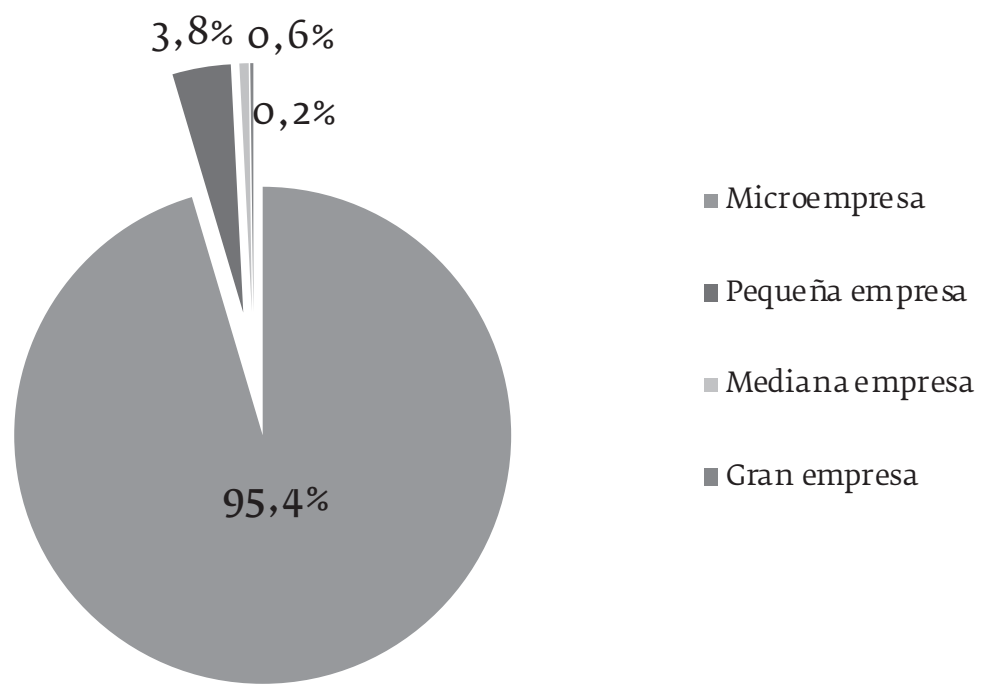

Figura 1: PyME de acuerdo al número de trabajadores

El número de empresas que forman parte de encontraba aleatoriamente distribuida, ello no esta investigación es 485.133 , ya que poseen información de ambos criterios para clasificar por tamaño a las empresas: número de trabajadores y ventas. Dicha cifra representa un 95\% del total de empresas censadas. La pérdida de información se implica la incorporación de sesgos. Como primer paso, para obtener un panorama general de la categorización de las empresas se analiza la distribución de las pequeñas y medianas empresas tomando en cuenta los dos criterios mencionados. 


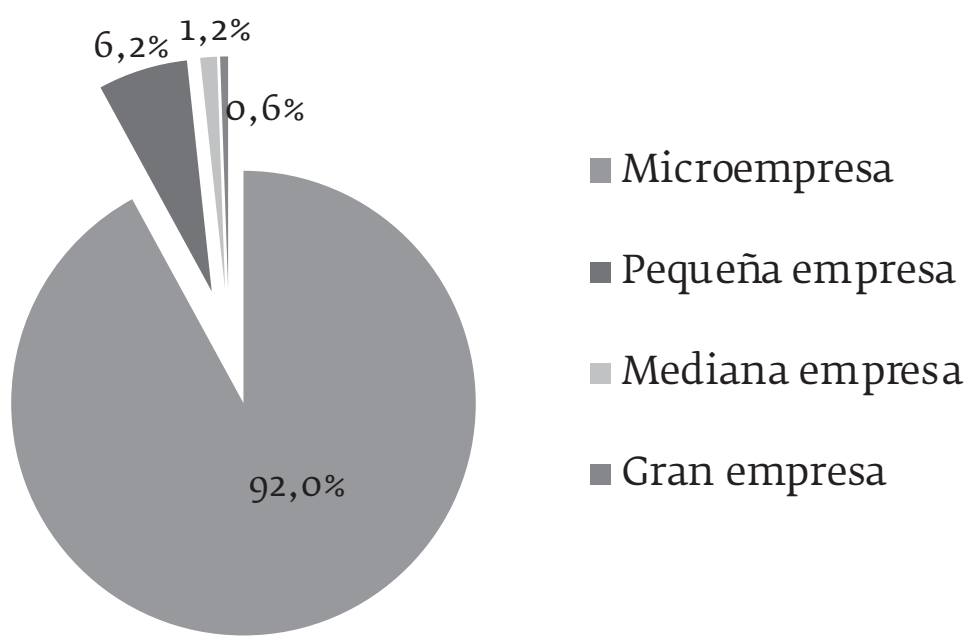

Figura 2 PyME de acuerdo al valor bruto de ventas

Categorías de empresas tos, las microempresas por número de trabajadores representan cerca de 20.000 empresas más

En la tabla 2 de doble entrada, se aprecia que existen diferencias entre las categorías de empresas debido al criterio tomado en cuenta; por ejemplo, en números absoluque al ser clasificadas por ventas brutas anuales, pero, a su vez, son solo alrededor de la mitad en pequeña, mediana y gran empresa.

Tabla 2 Categorización de las empresas por número de trabajadores y por ventas

Empresas por ventas brutas anuales

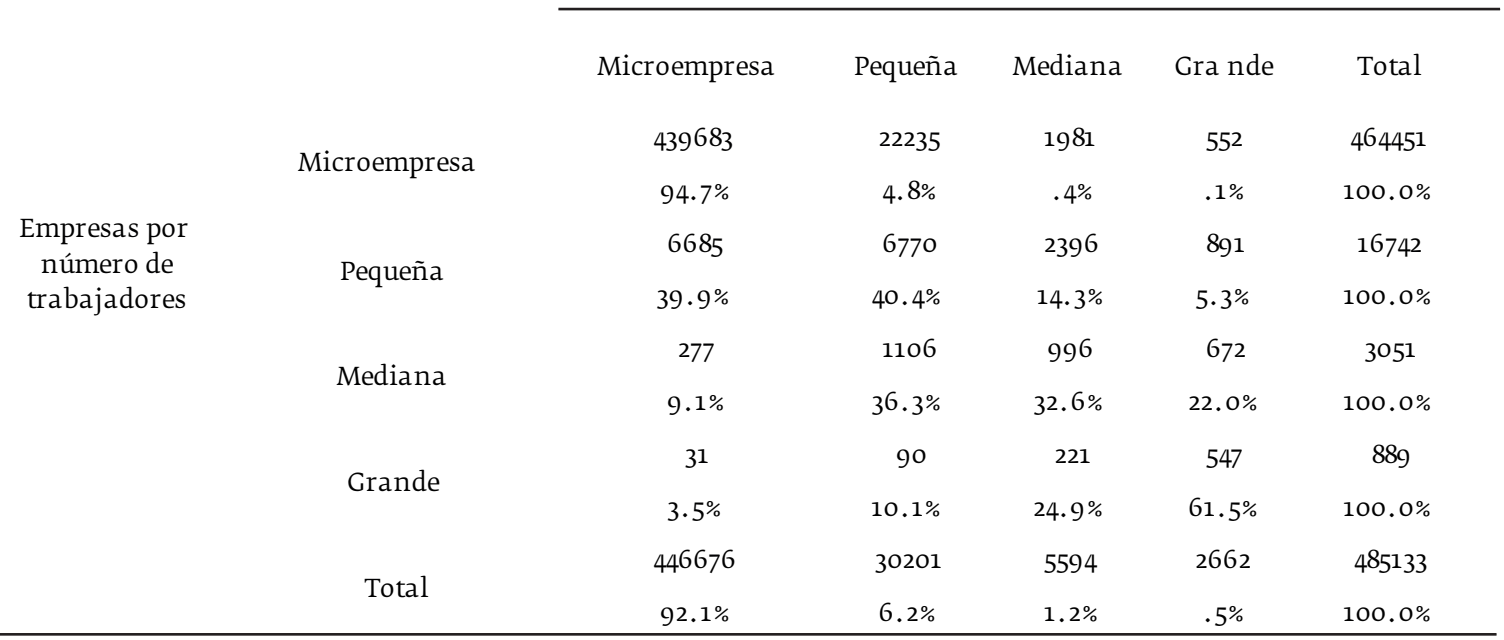

La corresponsabilidad entre categorías sería perfecta si solo existieran valores en la diagonal principal de la matriz, es decir si fuera una matriz diagonal, los cuales debieran coincidir con el total de forma vertical y horizontal. Como se observa, claramente este no es el caso, y a pesar de que hay correlación y dependencia entre criterios, a su vez, existen diferencias y asimetría entre grupos.

\section{Clasificación de empresas}

En la tabla 3 se presenta la clasificación de las empresas a nivel sectorial de acuerdo a ambos criterios. Existe heterogeneidad entre las micro, pequeñas y medianas empresas. Por un lado, entre las microempresas el comercio es el principal sector económico en ambos criterios con $55 \%$. Por el contrario, en las pequeñas empresas existe diferencias entre criterios, por el número de trabajadores el principal sector económico es servicios con cerca de $62 \%$, mientras que por ventas brutas anuales es comercio con $56 \%$. De igual manera, en las medianas empresas por el número de trabajadores el principal sector económico es servicios con cerca de $68 \%$, mientras que por ventas brutas anuales es comercio con $51 \%$. 
Tabla 3: Empresas por sector, categorizadas por número de trabajadores y por ventas

\begin{tabular}{|c|c|c|c|c|c|c|c|c|}
\hline & \multicolumn{4}{|c|}{ Empresas por número de trabajadores } & \multicolumn{4}{|c|}{ Empresas por ventas brutas anuales } \\
\hline & Micro & Pequeña & Mediana & Grande & Micro & Pequeña & Mediana & Grande \\
\hline Manufactura & $9.6 \%$ & $11.6 \%$ & $14.0 \%$ & $18.8 \%$ & $9.7 \%$ & $9.9 \%$ & $10.5 \%$ & $17 \cdot 3 \%$ \\
\hline Comercio & $56.3 \%$ & $25.1 \%$ & $14.9 \%$ & 11. $2 \%$ & $55.0 \%$ & $55.7 \%$ & $50.8 \%$ & $45.8 \%$ \\
\hline Servicios & $33.9 \%$ & $62.1 \%$ & $68.0 \%$ & $64.5 \%$ & $35.2 \%$ & $33.6 \%$ & $36.4 \%$ & $34.3 \%$ \\
\hline Total & $100.0 \%$ & $100.0 \%$ & $100.0 \%$ & $100.0 \%$ & $100.0 \%$ & $100.0 \%$ & $100.0 \%$ & $100.0 \%$ \\
\hline
\end{tabular}

En la tabla 4 se muestra que a nivel regional existen particularidades, aunque se mantiene la tendencia nacional de heterogeneidad entre empresas de acuerdo al criterio y tamaño. Tanto en la Sierra como en la Costa las características de las empresas se conservan en relación con lo nacional, pero hay cambios en la Amazonía y región Insular. Por un lado, en la Amazonia, tanto para la pequeña como la mediana y gran empresa, los servicios son la actividad más importante independientemente del criterio. Esto se explica principalmente debido a la presencia de empresas de servicio ligadas a la extracción de recursos naturales. Por otro lado, en la región Insular las empresas de manufactura y comercio, medidas por ambos criterios, reducen su presencia ante el sector servicios, y la respuesta a este comportamiento radica en la importancia del turismo.

Tabla 4 Empresas por región y sector, categorizadas por número de trabajadores y por ventas

\begin{tabular}{|c|c|c|c|c|c|c|c|c|c|}
\hline & & \multicolumn{4}{|c|}{ Empresas por número de trabajadores } & \multicolumn{4}{|c|}{ Empresas por ventas brutas anuales } \\
\hline & & Micro & Pequeña & Mediana & Grande & Micro & Pequeña & Mediana & Grande \\
\hline & Manufactura & $10.9 \%$ & $14.3 \%$ & $16.2 \%$ & $19.1 \%$ & $11.0 \%$ & $12.4 \%$ & $11.6 \%$ & $16.8 \%$ \\
\hline \multirow{4}{*}{$\begin{array}{l}\text { Región } \\
\text { Sierra }\end{array}$} & Comercio & $53 \cdot 5 \%$ & $24.4 \%$ & $14.0 \%$ & $10.0 \%$ & $52.3 \%$ & $51.3 \%$ & $49 \cdot 3 \%$ & $44.6 \%$ \\
\hline & Servicios & $35.4 \%$ & $60.4 \%$ & $67.0 \%$ & $64.4 \%$ & $36.5 \%$ & $35.8 \%$ & $37.4 \%$ & $35.7 \%$ \\
\hline & Otros & $.2 \%$ & $.8 \%$ & $2.8 \%$ & $6.5 \%$ & $.2 \%$ & $.5 \%$ & $1.6 \%$ & $2.8 \%$ \\
\hline & Manufa ctura & $8.1 \%$ & $8.7 \%$ & $12.0 \%$ & $19 \cdot 3 \%$ & $8.1 \%$ & $7.3 \%$ & $9 \cdot 5 \%$ & $18.5 \%$ \\
\hline \multirow{4}{*}{$\begin{array}{c}\text { Región } \\
\text { Costa }\end{array}$} & Comercio & $60.1 \%$ & $27.4 \%$ & $17.6 \%$ & $13.5 \%$ & $58.6 \%$ & $61.2 \%$ & $53.4 \%$ & $48.2 \%$ \\
\hline & Servicios & $31.7 \%$ & $62.0 \%$ & $66.7 \%$ & $62.8 \%$ & $33.2 \%$ & $30.3 \%$ & $33.7 \%$ & $31.1 \%$ \\
\hline & Otros & $.1 \%$ & $1.9 \%$ & $3.7 \%$ & $4.5 \%$ & $.1 \%$ & $1.2 \%$ & $3.4 \%$ & $2.3 \%$ \\
\hline & Manufactura & $9.1 \%$ & $4.0 \%$ & $4.1 \%$ & $0.0 \%$ & $9.3 \%$ & $4.3 \%$ & $3.1 \%$ & $2.9 \%$ \\
\hline \multirow{4}{*}{$\begin{array}{c}\text { Región } \\
\text { Amazónica }\end{array}$} & Comercio & $54.7 \%$ & $10.4 \%$ & $1.6 \%$ & $0.0 \%$ & $52.8 \%$ & $60.0 \%$ & $47 \cdot 9 \%$ & $25.7 \%$ \\
\hline & Servicios & $35.9 \%$ & $84.6 \%$ & $92.7 \%$ & $100.0 \%$ & $37.7 \%$ & $35.1 \%$ & $49.1 \%$ & $68.6 \%$ \\
\hline & Otros & $.3 \%$ & $1.0 \%$ & $1.6 \%$ & $0.0 \%$ & $.3 \%$ & $.7 \%$ & $0.0 \%$ & $2.9 \%$ \\
\hline & Manufactura & $7.5 \%$ & $0.0 \%$ & $0.0 \%$ & $0.0 \%$ & $6.8 \%$ & $10.1 \%$ & $0.0 \%$ & $0.0 \%$ \\
\hline \multirow{3}{*}{$\begin{array}{l}\text { Región } \\
\text { Insular }\end{array}$} & Comercio & $45.0 \%$ & $10.6 \%$ & $0.0 \%$ & $0.0 \%$ & $43.9 \%$ & $38.1 \%$ & $29.4 \%$ & $0.0 \%$ \\
\hline & Servicios & $47.4 \%$ & $89.4 \%$ & $100.0 \%$ & $100.0 \%$ & $49.2 \%$ & $51.8 \%$ & $70.6 \%$ & $100.0 \%$ \\
\hline & Otros & $.1 \%$ & $0.0 \%$ & $0.0 \%$ & $0.0 \%$ & $.1 \%$ & $0.0 \%$ & $0.0 \%$ & $0.0 \%$ \\
\hline
\end{tabular}

A nivel provincial también existen diferencias entre criterios. Las principales discrepancias están en los porcentajes de pequeñas y medianas empresas en servicios y comercio. Sin embargo, a su vez, hay tendencias similares, por ejemplo: independientemente del criterio, las microempresas en todas las provincias a excepción de Galápagos se dedican en un cincuenta por ciento o más al comercio. Además, Tungurahua es la provincia que mayoritariamente se dedica a la manufactura en las pequeñas y medianas empresas, aunque los valores son superiores (cerca del doble) al ser medidos por número de trabajadores.

\section{Características administrativas}

En relación con las características administrativas hay particularidades por sector, como se indica en la tabla 5. Las microempresas de manufactura se componen de un $75 \%$ de empresas creadas a partir del año 200o. El gerente es hombre en 3 de cada 4 empresas. Cerca de 
dos tercios tienen RUC y menos del 10\% llevan registros contables, realizan capacitación o usan internet. En lo referente a las pequeñas empresas manufactureras, por ventas, una de cada dos empresas ha sido creada desde el año 2000 , tienen registro contable, y usa internet. Además, cerca del 97\% tiene RUC. Con relación a las medianas empresas, cerca del $40 \%$ han sido creadas a partir del año 2000, y más del
$90 \%$ tienen registros contables y RUC. En comercio, en general las empresas son relativamente más nuevas que en manufactura, tienen gerentes hombres en menor proporción y realizan menos capacitación. En servicios, las PYME cumplen los requisitos legales como RUC y registros contables en mayor proporción que los otros sectores.

Tabla 5: Empresas por sector y características administrativas, categorizadas por número de trabajadores y por ventas

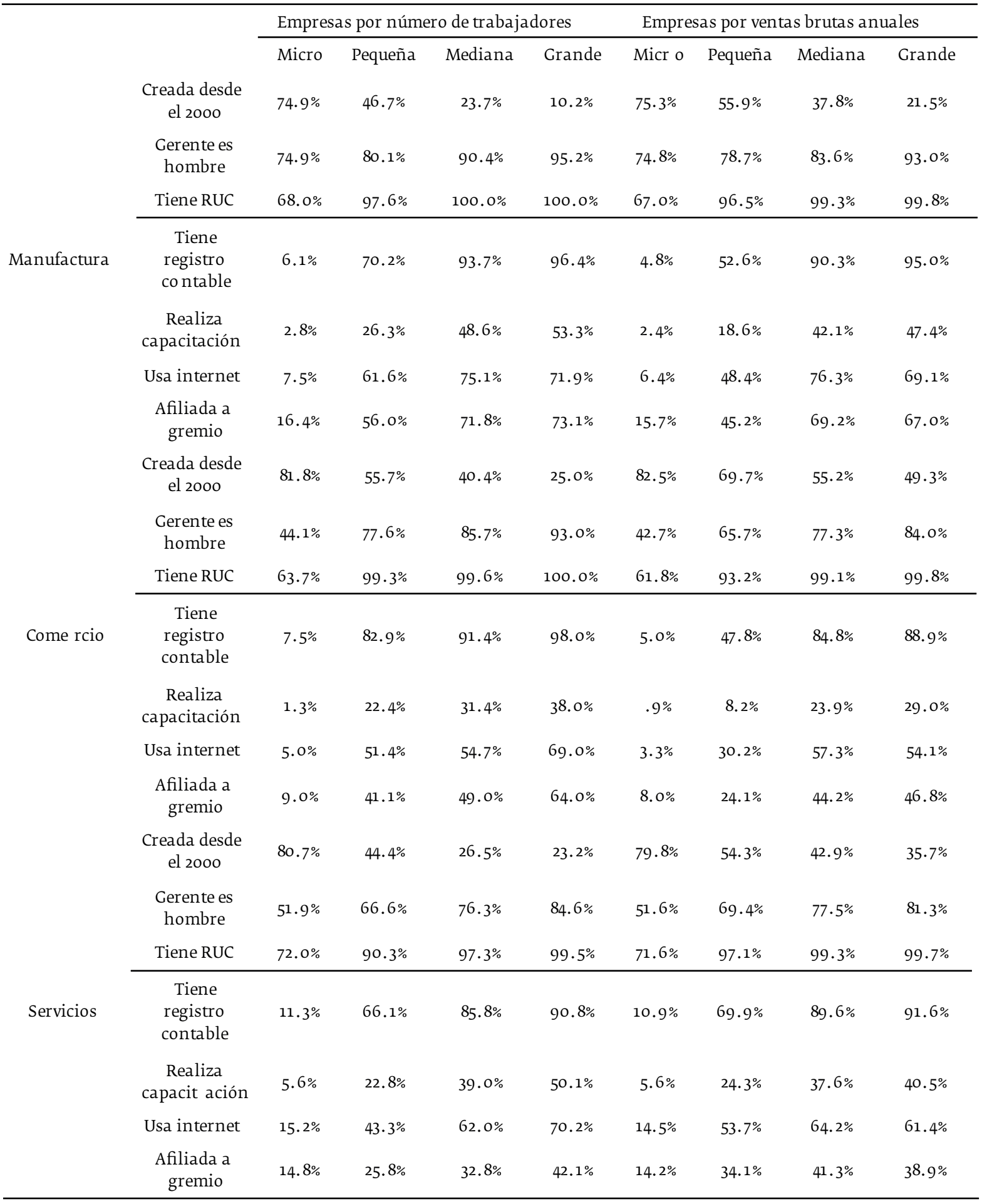




\section{Análisis probabilístico}

En cuanto al modelo probabilístico, los resultados se presentan en la tabla 6. Primero se aprecia que en general existen coeficientes estadísticamente significativos aunque su magnitud no es alta. Adicionalmente, lo encontrado se corresponde con lo mencionado en la primera parte sobre correlaciones. Las variables geográficas de la región Sierray Amazonía no resultan ser significativas, y solo la Costa es representativa con signo negativo; es decir, las empresas costeñas tienen un $0.03 \%$ menos probabilidad de ser PyME que aquellas ubicadas en la región Insular.

En referencia a las variables sectoriales, estas resultan significativas y nos indican que pertenecer al sector comercio y servicios aumenta la probabilidad de ser PyMEen $0.01 \%$ y $0.04 \%$ en relación con pertenecer al sector manufacturero. Esto sucede ya que las grandes empresas tienen mayor representación en el sector manufacturero.En ese sentido, el sector en donde se desempeñan las PyME es relevante (Motta, 2017).

Por último, con relación a las características administrativas, estas resultaron ser significativas y en su mayoría tienen efecto negativo en la probabilidad de ser PYME. Por ejemplo, tener RUC reduce la probabilidad en $0.08 \%$, el llevar registros contables afecta en $0.9 \%$, gastar en capacitación reduce en $0.1 \%$, el uso de internet en $0.03 \%$, el afiliarse a gremios en $0.01 \%$, tener gerente masculino en $0.04 \%$.

Ante ello, se podría afirmar que a medida que la empresa formaliza sus operaciones se incrementa su probabilidad de ser gran empresa antes que PyME. Solamente la característica que se refiere a la creación de la empresa a partir del año 2000 aumenta la probabilidad de ser PyME en $0.05 \%$. Este resultado también es lógico debido a que llegar a ser una gran empresa toma tiempo.

Tabla 6: Determinantes de las micro, pequeña y mediana empresa -PyME -

\begin{tabular}{|c|c|c|}
\hline & \multicolumn{2}{|c|}{ PyME } \\
\hline \multicolumn{3}{|l|}{ Características geográficas } \\
\hline Región Sierra & $\begin{array}{l}-0.0001 \\
(0.00)\end{array}$ & \\
\hline Región Costa & $\begin{array}{l}-0.0003 \\
(0.00)\end{array}$ & * \\
\hline Región Amazonía & $\begin{array}{l}-0.0001 \\
(0.00)\end{array}$ & \\
\hline \multicolumn{3}{|l|}{ Características sectoriales } \\
\hline Comercio & $\begin{array}{l}0.0001 \\
(0.00)\end{array}$ & *** \\
\hline Servicios & $\begin{array}{l}0.0004 \\
(0.00) \\
\end{array}$ & $* * *$ \\
\hline \multicolumn{3}{|l|}{ Características administrativas } \\
\hline Posee RUC & $\begin{array}{l}-0.0008 \\
(0.00)\end{array}$ & $* * *$ \\
\hline Tiene registros contables & $\begin{array}{l}-0.009 \\
(0.00)\end{array}$ & $* * *$ \\
\hline Gasta en capacitación & $\begin{array}{l}-0.001 \\
(0.00)\end{array}$ & $* * *$ \\
\hline Usa internet & $\begin{array}{l}-0.0003 \\
(0.00)\end{array}$ & $* * *$ \\
\hline Afiliado a gremios & $\begin{array}{l}-0.0001 \\
(0.00)\end{array}$ & $* * *$ \\
\hline Creadas desde el año 2000 & $\begin{array}{l}0.0005 \\
(0.00)\end{array}$ & $* * *$ \\
\hline Gerente masculino & $\begin{array}{l}-0.0004 \\
(0.00)\end{array}$ & $* * *$ \\
\hline $\mathrm{N}$ & 485.133 & \\
\hline Probabilidad correctamente predicha & 99.45 & \\
\hline
\end{tabular}

Nota:La variable dependiente es binaria y toma el valor de 1 si la empresa es PyME. El reporte corresponde a los coeficientes marginales, obtenidos con dprobit. Nivel de significancia: *** $0.01,{ }^{* *} 0.05,{ }^{*} 0.1$. 


\section{Discusión}

Las PyME abarcan poco menos de la totalidad del sector empresarial ecuatoriano. El presente estudio pretende ser un aporte en tresaspectos principales. Primero, mediante la revisión de la literatura sobre la definición de PyME, se encuentra que este concepto tiene varias aristas y hay un debate en torno a los criterios utilizados. La definición estándar de PyME está dando paso a nuevas concepciones, diversas, que incorporan elementos adicionales.

Segundo, en relación con los criterios empleados en Ecuador para clasificar a las empresas por número de trabajadores y ventas, se encuentra que hay diferencias entre ambos métodos, aunque se hallen relacionados. El contraste de Pearson indica que ambas medidas son dependientes entre sí, con diferentes medias y desviaciones estándar. A su vez, las diferencias se incrementan al descomponer el análisis por las variables geográficas, sectoriales y administrativas. En ese sentido, existe correspondencia con lo encontrado en el estudio de Briozzo, Vigier y Martinez (2016) donde existen elementos adicionales a la normativa que determinan a las PyME.

Tercero, en referencia a los resultados del modelo probabilístico se puede expresar que pertenecer al sector comercio y servicios aumenta la probabilidad de ser PyME. Este resultado se explica debido a que las grandes empresas se presentan en mayor proporción en el sector de manufactura. Por el contrario, formalizar la parte administrativa, con RUC y registros administrativos, reduce su probabilidad. Este resultado tiene su razón en que a medida que aumenta el tamaño de la empresa, se incrementan las ventas, se establecen procesos administrativos, y se extiende la consecuente división del trabajo. Una desventaja evidente de la ausencia de formalidad en las PyME es que pierden oportunidades de acceso a crédito en el sistema financiero y capacitación (García y Villafuerte, 2015); donde existen barreras de oferta y demanda (Kumar y Rao, 2015). Asimismo, el fortalecer a la PyME puede ayudar a mejorar el proceso de internacionalización de las mismas (Araque y García, 2015).

\section{Conclusiones}

La definición de PyME en Ecuador se compone de dos elementos: número de trabajadores y ventas. En este estudio se encuentra que no necesariamente se obtienen los mismos resultados usando un solo criterio. Dichas diferencias se relacionan con la heterogeneidad del sector. Además, existen determinantes de las PyME relacionadas con las características geográficas, sectoriales y administrativas. Con ello, las políticas públicas enfocadas en mejorar el desarrollo de las micro, pequeñas y medianas empresas debe propender a un análisis focalizado por sector, es decir, tomando en cuenta sus particularidades y contextualizando sus características.

\section{Referencias}

Alvarado, R., Peñarreta, M., Armas, R., \& Alvarado, R. (2017). Access to financing and regional entrepreneurship in Ecuador: an approach using spatial methods. International Journal of Entrepreneurship 21(3): 1-9.

Araque, W. (2018). Asociatividad en la PyME. Quito: Universidad Andina Simón Bolívar.

Araque, W. \&García, S. (2015). Oportunidades de exportación para las PyME en mercados latinoamericanos. Quito: Universidad Andina Simón Bolívar.

Araque, W. \& Argüello A. (2015). Caracterización del proceso de internacionalización de las PyME ecuatorianas. FAEDPYME International Review 4(7):35-46.

Ayyagari, M., Demirgüc-Kunt, A., \&Maksimovic, V. (2011). Small and Medium Enterprises Across the Clobe: Contribution to Employment, Job Creation, and Growth. World Bank Policy Research Working Paper 5631. Washington: World Bank.

Ayyagari, M., Thorsten B., \&Demirgüc-Kunt, A. (2005). Small and Medium Enterprises Across the Globe. World Bank Policy Research Working Paper 3127.Washington: World Bank.

Asamblea Nacional. (2010). Código Orgánico de la Producción, Comercio e Inversiones, COPCI. Quito: Asamblea Nacional.

Briozzo, A., Vigier, H., \& Martinez, L. (2016). Firm-Level Determinants of the Financing Decisions of Small and Medium Enterprises: Evidence from Argentina. Latin American Business Review, 17(3): 245-268. doi:10.1080/10978526.2016 .1209081

Comunidad Andina de Naciones (CAN). (2008). Decisión 702: Sistema Andino de Estadística de la PyME. Lima: CAN.

European Union. (2015). User guide to the SME definition. Belgium: EU.

García, G. \& Villafuerte, M. (2015). Las restricciones al financiamiento de las PYMES. Actualidad Contable Actualidad Contable FACES 30: 49-73.

García, S. (2012). Caracterización y situación actual de PYME exportadoras ecuatorianas en los mercados de ALADI. Quito: ALADI-Observatorio de la PYME.

Gibson, T. \& Van der Vaart H. (2008). Defining SMEs: A Less Imperfect Way of Defining Small and Medium Enterprises in Developing Countries. Washington: The Brookings Institution.

Instituto Nacional de Estadísticas y Censos (INEC). (2010). Censo Nacional Económico. Quito: INEC.

INEC. (2011) Avances del Censo Nacional Económico y Mecanismos de Difusión. Quito: INEC.

INEC. (2013). Directorio de Empresas y Establecimientos. Quito: INEC.

Jácome, H. \&King, K. (2012). Estudios industriales de la micro, pequeña y mediana empresa. Quito: FLACSO.

Kumar, S., \&Rao, P. (2015). A conceptual framework for identifying financing preferences of SMEs. Small Enterprise Research, 22(1), 99-112. doi:10.1 080/13215906.2015.1036504 
Massón-Guerra, J.L. \&Ortín-Ángel, P. Small Bus Econ (2019) 52: 175. https://doi.org/10.1007/ s11187-018-0014-8

Motta, Victor. (2017). Are SMEs in the hospitality industry less likely to experience credit constrain tan other industries in the service sector? Evidence from Latin America. Tourism Economics 23(7): 1398-1418.

Mercado Común del Sur. (1993). Resolución № 9o/93. Políticas de apoyo a las micro, pequeñas y medianas empresas del MERCOSUR. Asunción: MERCOSUR.

Organisation for Economic Co-operation and Development (OECD). (2007). Glosary of Statistical Terms. Paris: OECD.

OECD. (2005a). OECD SME and Entrepreneurship Outlook. Paris: OECD.

OECD. (2005b). SBS Expert Meeting “Towards better Structural Business and SME Statistics. Paris: OECD

Peres, W., \&Stumpo, G. (2000). Small and Medium-Sized Manufacturing Enterprises in Latin
America and the Caribbean Under the New Economic Model. WorldDevelopment, 28(9): 16431655. doi:10.1016/s0305-750x(oo)0oo46-2

Presidencia de la República. (2011). Reglamento a la estructura e institucionalidad de desarrollo productivo, de la inversión y de los mecanismos e instrumentos de fomento productivo establecidos en el COPCI. Quito: Presidencia de la República.

Sarango-Lalangui, P.; Álvarez-García, J.; Del Río-Rama, M. (2018). Sustainable Practices in Small and Medium-Sized Enterprises in Ecuador. Sustainability 2018, 10, 2105. https://doi. org/10.3390/su10062105

Saavedra, M. \&Hernández, Y. (2008). Caracterización e importancia de las MIPYMES en Latinoamérica. ActualidadContable FACES 10: 122-134.

Small Business Administration (SBA). (2016). Table of Small Business Size Standards Matched to North American Industry Classification System Codes. Washington: SBA.

Wooldridge, J. (2013). Introductory Econometrics: a modern approach. Michigan: Cengage Learning. 